\title{
Ultrasound-assisted Synthesis of Biodiesel from Crude Cottonseed Oil Using Response Surface Methodology
}

\author{
Xiaohu Fan ${ }^{1}$, Feng Chen ${ }^{1}$ and Xi Wang*2 \\ ${ }^{1}$ Department of Food Science and Human Nutrition, Clemson University (Clemson, SC 29634, USA) \\ ${ }^{2}$ Department of Genetics and Biochemistry, Clemson University (Clemson, SC 29634, USA)
}

\begin{abstract}
Response surface methodology (RSM) based on central composite rotatable design (CCRD) was used to optimize the three important reaction variables-methanol/oil molar ratio $(M)$, NaOH catalyst concentration $(C)$ and reaction time $(T)$ for transesterification of crude cottonseed oil under ultrasonic irradiation. A quadratic polynomial model was obtained to predict the methyl ester yield. $98 \mathrm{wt} \%$ of the methyl ester yield could be reached at the deduced optimal conditions: methanol/oil molar raito of 6.2:1, $\mathrm{NaOH}$ catalyst concentration of $1 \mathrm{wt} \%$ (by the weight of crude cottonseed oil) and reaction time of 8 min. Validation experiments confirmed the validity of the predicted model. Moreover, ultrasonic irradiation was proved to be an efficient, energy saving and economically feasible way to produce biodiesel.
\end{abstract}

Key words: biodiesel, central composite rotatable design (CCRD), crude cottonseed oil, response surface methodology (RSM), ultrasonic irradiation

\section{INTRODUCTION}

Biodiesel is currently of interest due to high energy demand, the limited resource of fossil fuel and environmental concerns. Made from vegetable oil, such as cottonseed oil, soybean oil, rapeseed oil or animal fat, biodiesel is a renewable, biodegradable, non-toxic and clean-burning fuel ${ }^{1-3)}$, producing favorable effects on the environment ${ }^{4,5)}$.

The most common method for producing this fuel is transesterification, a chemical process in which an alcohol, usually methanol, reacts with triglycerides to generate biodiesel and the by-product, glycerol in the presence of catalyst, usually alkaline (sodium hydroxide or potassium hydroxide) when the free fatty acid content in the raw material is below $1 \mathrm{wt} \%$.

To obtain increased biodiesel yields within shorter reaction times, ultrasonically-assisted production of biodiesel is currently the focus of new research ${ }^{6-9)}$. This process improve the mass transfer between two immiscible liquids, methanol and oils through cavitation, the phenomenon in which bubbles containing primary vapor reduce the ambient pressure sufficiently at essentially constant temperatures, causing an explosive vaporization into the cavities. Stavarache et al. ${ }^{10)}$ found the efficient mixing problems could be overcome by using low frequency ( $28 \mathrm{kHz}$ and 40 $\mathrm{kHz}$ ) ultrasounds when transesterifying commercially edible grade vegetable oil with short-chain alcohols in the presence of base catalyst. They concluded that supersonic jets are created during the collapse of cavitational bubbles of methanol, generating nano-sized drops that are extremely efficiently mixed thus abundantly enhancing the reaction surface ${ }^{11)}$.

The advantages of applying ultrasonic methods to biodiesel production, such as enhancement of mixing efficiency, less energy consumption etc., are well-known. Similar to the conventional method, variables such as the methanol/oil molar ratio, reaction time, and the catalyst concentration have a significant effect on the biodiesel yield. To determine the optimal condition, Hanh et al. ${ }^{12)}$ evaluated these variables by transesterifying triolein with ethanol under ultrasonic irradiation. The optimal conditions were then identified. However, the process was timeconsuming and complex since the interrelationships among the test variables have to be determined.

Response surface methodology (RSM), a powerful tool in the optimization of chemical reactions, addresses this issue by providing 1 . an understanding of how the test vari-

\footnotetext{
* Correspondence to: Xi Wang, Department of Genetics and Biochemistry, Clemson University, Clemson, SC 29634, USA

E-mail: xiwclemson@gmail.com

Accepted December 21, 2009 (received for review October 17, 2009)
}

Journal of Oleo Science ISSN 1345-8957 print / ISSN 1347-3352 online

http://www.jstage.jst.go.jp/browse/jos/ 
ables affect the selected process response; 2 . the determination of the interrelationships among the test variables; and 3. the characterization of the combined effect that all influential test variables may have on the process response. Because of these advantages, RSM has been increasingly applied to transesterification in biodiesel production. For example, Ghadge and Raheman ${ }^{13)}$ used this methodology to optimize the pretreatment process for reducing the free fatty acid (FFA) content of mahua oil to below $1 \mathrm{wt} \%$ for maximum biodiesel production. Similarly, Tiwari et al. ${ }^{14)}$ obtained quadratic polynomial models using RSM to predict acid value of the jatropha oil (Jatropha curcas) before its conversion to biodiesel. Li et al. ${ }^{15)}$ applied the same method developing a polynomial model predicting the yield of biodiesel when utilizing whole cell biocatalyst. Most importantly, RSM is the preferred method for optimizing biodiesel production from waste rapeseed oil with high FFA $^{16)}$.

However, little research has been conducted using cottonseed oil in the production of biodiesel using RSM, especially in the case of ultrasonic-assisted process, since this oil contains natural pigments (such as gossypol) that can enhance the stability of the fuel. To address this issue, the study reported here investigated the ultrasonically assisted production of biodiesel (methyl ester in this experiment) from crude cottonseed oil. The primary objective was to evaluate the suitability of RSM for optimizing the methanolysis of crude cottonseed oil, including the development of a mathematical model describing the relationships and subsequent effects of the primary process variables.

\section{EXPERIMENTAL}

\subsection{Materials and reagents}

Methanol was purchased from Fisher Scientific (Suwanee, GA, USA). Sodium hydroxide was bought from Sigma chemical company (St. Louis, MO, USA). Crude cottonseed oil derived from expeller, i.e. screw pressed cottonseed, was obtained from the Elgin Cotton Oil Mill, Inc. (Elgin, TX, USA).

\subsection{Experimental design}

"A 3-factor experiment was conducted using a central composite rotatable design (CCRD) to examine effects of methanol/oil molar ratio, reaction time, and catalyst concentration on yield of methyl ester." The CCRD consisted of 20 experimental runs (eight factorial points, eight axial points, and four replicated center points) and provided sufficient information to fit a full second-order polynomial model. Results from previous research ${ }^{16)}$ were used to establish the center point of the CCRD for each factor: 6/1, $1 \mathrm{wt} \%$, and $8 \mathrm{~min}$ for methanol/oil molar ratio, $\mathrm{NaOH}$ catalyst concentration, and reaction time, respectively. Table 1 provides the levels used for each factor, and to avoid bias, the 20 experimental runs were performed in randomized order (Table 2).

\subsection{Transesterification of crude cottonseed oil}

The ultrasonic reaction system is comprised of ultrasound reactor, power supply amplifier (Model G 7520), and function generator (Model 182A, $4 \mathrm{MHz}$ ), which converts a standard line voltage to a high-frequency electrical power. This electrical energy fed to the transducer, which is inside the soundproof enclosure and has a total power of $600 \mathrm{~W}$, can be converted to mechanical vibrations of the same frequency.

Transesterification of crude cottonseed oil with methanol in the presence of $\mathrm{NaOH}$ proceeded at room temperature $\left(25^{\circ} \mathrm{C}\right)$ under $40 \mathrm{kHz}$ ultrasonic irradiation. The amount of methanol needed was determined by the methanol/oil molar ratio. An appropriate amount of $\mathrm{NaOH}$ catalyst dissolved in the methanol was added to the precisely prepared crude cottonseed oil (40 $\pm 0.1 \mathrm{~g})$. This mixture was then introduced to the ultrasound reactor. After ultrasonic irradiation, the reaction mixture was neutralized immediately with phosphoric acid solution to stop further reaction. The mixture was then transferred to a separatory funnel and allowed to stand for phase separation. After the phase separation, the methanol and traces of catalyst were washed out with water from the ester mixture until the water layer remained completely translucent. Finally, the ester layer was dried and analyzed.

Table 1 Independent Variable and Levels Used for CCRD in Methyl Ester Production.

\begin{tabular}{lcccccc}
\hline \multicolumn{1}{c}{ Variables } & Symbols & \multicolumn{5}{c}{ Levels $^{\mathrm{a}}$} \\
\cline { 3 - 7 } & & -1.682 & -1 & 0 & +1 & +1.682 \\
\hline Reaction time (min) & $\mathrm{T}$ & 0.43 & 3.5 & 8.0 & 12.5 & 15.57 \\
Methanol/oil molar ratio (mol/mol) & $\mathrm{M}$ & 0.95 & 3 & 6 & 9 & 11 \\
Catalyst concentration (wt\%) & $\mathrm{C}$ & 0.16 & 0.5 & 1 & 1.5 & 1.8 \\
\hline
\end{tabular}

${ }^{\mathrm{a}}$ Transformation of variable levels from coded $(\mathrm{X})$ to uncoded could be obtained as: $\mathrm{T}=8.0+4.5 \mathrm{X}$, $\mathrm{M}=6+3 \mathrm{X}$ and $\mathrm{C}=1+0.5 \mathrm{X} .(-1.682,-1,0,+1$, and +1.682 are coded values. $)$ 
Ultrasound-assisted Synthesis of Biodiesel from Crude Cottonseed Oil

Table 2 CCRD Arrangement and Responses for Methyl Ester Production.

\begin{tabular}{rrrcrcc}
\hline Treatment & Random & \multicolumn{3}{c}{ Level of variables } & \multicolumn{2}{c}{$\begin{array}{c}\text { Yield (\%) } \\
\text { (response) }\end{array}$} \\
\cline { 3 - 7 } & & T, min & M, mol/mol & C, wt $\%$ & Experimental & Predicted \\
\hline 1 & 8 & 12.5 & 9 & 1.5 & 95.5 & 97.3 \\
2 & 12 & 8 & 6 & 1 & 96.0 & 97.6 \\
3 & 7 & 3.5 & 9 & 1.5 & 73.3 & 84.9 \\
4 & 14 & 15.6 & 6 & 1 & 98.4 & 96.3 \\
5 & 2 & 12.5 & 3 & 0.5 & 23.9 & 15.5 \\
6 & 19 & 8 & 6 & 1 & 97.6 & 97.6 \\
7 & 1 & 3.5 & 3 & 0.5 & 22.9 & 24.3 \\
8 & 9 & 8 & 6 & 1 & 98.0 & 97.6 \\
9 & 20 & 8 & 6 & 1 & 98.0 & 97.6 \\
10 & 13 & 0.43 & 6 & 1 & 97.0 & 93.2 \\
11 & 6 & 12.5 & 3 & 1.5 & 41.7 & 51.7 \\
12 & 4 & 12.5 & 9 & 0.5 & 86.8 & 87.8 \\
13 & 18 & 8 & 6 & 1.8 & 91.0 & 75.7 \\
14 & 3 & 3.5 & 9 & 0.5 & 76.8 & 70.1 \\
15 & 16 & 8 & 11 & 1 & 90.3 & 86.0 \\
16 & 5 & 3.5 & 3 & 1.5 & 63.6 & 65.8 \\
17 & 17 & 8 & 6 & 0.16 & 21.5 & 29.6 \\
18 & 10 & 8 & 6 & 1 & 97.0 & 97.6 \\
19 & 15 & 8 & 0.95 & 1 & 10.0 & 8.4 \\
20 & 11 & 8 & 6 & 1 & 98.0 & 97.6 \\
\hline
\end{tabular}

T: Reaction time, M: Methanol/oil molar ratio, C: Catalyst concentration

\subsection{Analytical methods}

Free fatty acid content of the crude cottonseed oil was measured according to A.O.C.S. Official Method Ca 5a$40^{177}$.

The Shimadzu'fs GC-FID system consisting of a GC-17A with a flame ionization detector was installed with a DBWAX capillary column $(60 \mathrm{~m} \times 0.25 \mathrm{~mm}$, thickness $=0.25$ $\mu \mathrm{m}$; J\&W Scientific) for fatty acid profile analysis of crude cottonseed oil. Two to three microliters of the sample was injected with the split ratio equal to 1 . The initial temperature for oven was $180^{\circ} \mathrm{C}$ and held for $2 \mathrm{~min}$, then the temperature was programmed from $180^{\circ} \mathrm{C}$ to $250^{\circ} \mathrm{C}$ at the ramp of $5{ }^{\circ} \mathrm{C} / \mathrm{min}$ and held at $250^{\circ} \mathrm{C}$ for half an hour. The injector and detector were set at $200^{\circ} \mathrm{C}$ and $220^{\circ} \mathrm{C}$, respectively. Helium was used as carrier gas and the column flow rate was $1.5 \mathrm{~mL} / \mathrm{min}$.

The methyl ester produced was evaluated by using reverse phase HPLC. The Shimadzu HPLC system consisted of an evaporative light scattering detector (ELSD) with a Phenomenex Gemini C18 column $(250 \times 4.6 \mathrm{~mm}, 5 \mu \mathrm{m})$.
HPLC grade acetonitrile (A) and dichloromethane (B) were selected as the mobile phase. The gradient program was as follows: Time:(0, 5, 30, $32 \mathrm{~min}) \mathrm{B}:(0,15,70,70 \%)$. The flow rate was $1.0 \mathrm{~mL} / \mathrm{min}$. Each time $10 \mu \mathrm{L}$ of diluted methyl ester was injected.

\subsection{Statistical analysis}

The experimental data presented in Table 2 was analyzed using the response surface regression (RSREG) procedure in SAS ${ }^{18)}$ that fits a full second-order polynomial model:

$$
y=\beta_{0}+\sum_{i=1}^{3} \beta_{i} x_{i}+\sum_{i=1}^{3} \beta_{i i} x_{i}^{2}+\sum_{i=1}^{3} \sum_{j=1}^{2} \beta_{i j} x_{i} x_{j}
$$

where $\mathrm{y}$ is $\mathrm{wt} \%$ methyl ester yield, $\mathrm{x}_{\mathrm{i}}$ and $\mathrm{x}_{\mathrm{j}}$ are the independent study factors, and $\beta_{0}, \beta_{\mathrm{i}}, \beta_{\mathrm{ii}}$, and $\beta_{\mathrm{ij}}$ are intercept, linear, quadratic, and interaction constant coefficients, respectively. An $\alpha$ level of 0.05 was used to examine the 
statistical significance of the fitted polynomial model. The RSREG procedure uses canonical analysis to estimate stationary values for each factor. Using the fitted model, response surface contour plots were constructed for each pair of study factors while holding the other factor constant at its estimated stationary point. Confirmatory experiments were carried out to validate the model using combinations of independent variables that were not a part of the original experimental design but within the experimental region.

\section{RESULTS AND DISCUSSION}

\subsection{Characterization of raw material}

GC analyses results indicated that the triglycerides found in this crude cottonseed oil contained $23.67 \mathrm{wt} \%$ of palmitic acid (16:0), $17.09 \mathrm{wt} \%$ of oleic acid (18:1), and 50.33 wt\% of linoleic acid (18:2) as their main fatty acid components. Since higher amount of free fatty acids (FFA) $(>1$ wt\%) in the feedstock can directly react with the alkaline catalyst to form soaps, which are subject to form stable emulsions and thus prevent separation of the biodiesel from the glycerol fraction ${ }^{19)}$, it is better to select reactant oils with low FFA content or to remove FFA from the oil to an acceptable level before the reaction. The FFA (calculated as oleic acid) content for the crude cottonseed oil used in this experiment was only $0.8 \mathrm{wt} \%$, it was suitable for transesterification reactions under direct alkaline catalysis.

\subsection{RSM analysis of transesterification}

Table 3 listed the regression coefficients and the corresponding $p$-values for the second-order polynomial model. It could be seen from the $p$-values of each model term that the regression coefficients of the linear term $M, C$, the quadratic term, $M^{2}, C^{2}$, and the interaction term, $T C, M C$ had significant effect on the yield ( $p$-value $<0.05$ ). Among them, $M, C, C^{2}, M C$ were significant at $1 \%$ level, while $M^{2}$
Table 3 Regression Coefficients of Predicted Quadratic Polynomial Model for Methyl Ester Production.

\begin{tabular}{ccc}
\hline Terms & Regression coefficients & $p$-value \\
\hline Intercept & & \\
$\beta_{0}$ & -123.93 & 0.0005 \\
Linear & & \\
$\beta_{1}$ & -1.36 & 0.6124 \\
$\beta_{2}$ & 32.02 & 0.0001 \\
$\beta_{3}$ & 188.97 & 0.0001 \\
Quadratic & & \\
$\beta_{11}$ & -0.05 & 0.6643 \\
$\beta_{22}$ & -1.99 & 0.0489 \\
$\beta_{33}$ & -66.06 & 0.0001 \\
Interaction & & \\
$\beta_{12}$ & 0.49 & 0.6611 \\
$\beta_{13}$ & -0.59 & 0.0482 \\
$\beta_{23}$ & -4.44 & 0.0001 \\
\hline
\end{tabular}

and $T C$ were significant at $5 \%$ level.

Using the coefficients determined (Table 3), the predicted model in terms of uncoded factors for methyl ester yield is:

$$
\begin{aligned}
Y_{\text {yield }}= & -123.93-1.36 T+32.02 M+188.97 C+0.49 T M- \\
& 0.59 T C-4.44 M C-0.05 T^{2}-1.99 M^{2}-66.06 C^{2}
\end{aligned}
$$

Where $Y_{\text {yield }}$ is the response, that is, the methyl ester yield, and $T, M$, and $C$ are the actual values of the test variables, reaction time, methanol/oil molar ratio, and catalyst

Table 4 Analysis of Variance (ANOVA) for the Quadratic Model.

\begin{tabular}{cccccc}
\hline Variance source & Sum of squares & Degrees of freedom & Mean square & $F$-value & $p$-value \\
\hline Regression & 18034.588 & 9 & 2003.843 & 28.569 & $<0.0001$ \\
Linear & 9709.719 & 3 & 3236.573 & 46.145 & $<0.0001$ \\
Quadratic & 7602.995 & 3 & 2534.332 & 36.133 & $<0.0001$ \\
Interaction & 721.874 & 3 & 240.625 & 3.431 & 0.0603 \\
Residual error & 701.397 & 10 & 70.140 & & \\
Total error & 18735.985 & 19 & & & \\
$\mathrm{R}^{2}=0.963$ & & & & & \\
\hline
\end{tabular}


concentration, respectively.

It could be concluded from Table 3 that the linear effects of $M, C$ and the quadratic effect of $C^{2}$ were the primary determining factors on the methyl ester yield as they had the largest coefficient. Meanwhile, the quadratic effect of $M^{2}$ and the interaction effect of $M C$ were the secondary determining factors with medium coefficient. Other terms of the model showed no significant effect on $Y_{\text {yield }}$. Among them, $M$ and $C$ had positive coefficient, exhibiting the enhancement on the yield. However, all the other terms had negative coefficient.

Table 4 showed the analysis of variance $(F$-test) and the $p$-value for this model. The $F$-value was 28.57 and the $p$ value was smaller than 0.0001 , demonstrating the suitability of the deduced model. The $R^{2}$ value $(=0.963)$ indicated that the quadratic model was able to predict $96.3 \%$ of the total variance and only $3.7 \%$ of the total variance was not explained by the model.

\subsection{Effect of parameters}

Contour plots (Fig. 1A-1C) were drawn to show the relationships between dependent and independent variables of the developed model. Each contour curve presented the effect of two variables on the methyl ester yield, holding the third variable at constant level. Remarkable interaction between the independent variables could be observed if the contour plots had an elliptical profile.

Figure $1 \mathrm{~A}$ showed the strong interaction between methanol/oil molar ratio $(M)$ and $\mathrm{NaOH}$ catalyst concentration $(C)$. This can also be confirmed by the small $p$-value (0.0001) for $M C$ term. It could also be seen from Fig. 1A that the methyl ester yield increased with increasing catalyst concentration at first. However, when the catalyst concentration reached a certain level, the reverse trend was observed. The similar pattern was followed when increasing methanol/oil molar ratio. This could be due to the fact that the positive coefficient for $C$ and $M$ played the main role when the $\mathrm{NaOH}$ catalyst concentration and methanol/ oil molar ratio were at lower level, while at higher level, the interaction term $M C$ and quadratic term $M^{2}$ and $C^{2}$ showed more significant negative effect, leading to the decrease of the yield. This was consistent with the physical explanation. Since the methanol and triglyceride in the crude cottonseed oil are immiscible, addition of catalyst can facilitate the transesterification reaction, and rapidly increase the yield. However, when the catalyst concentration was too high, soap could be quickly formed which made the separation of glycerol from biodiesel more difficult, thus reducing the yield. Similarly, the increase of the methanol amount, on one hand, will drive the reaction to the right since the transesterification reaction is an equilibrium process; on the other hand, excess methanol will help increase the solubility of glycerol resulting in the reaction driven to the left, thus decreasing the yield.

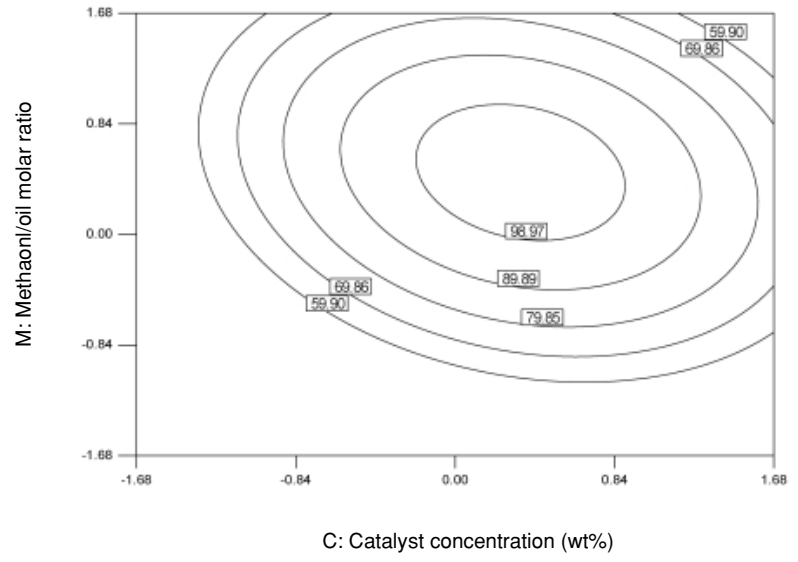

(A)

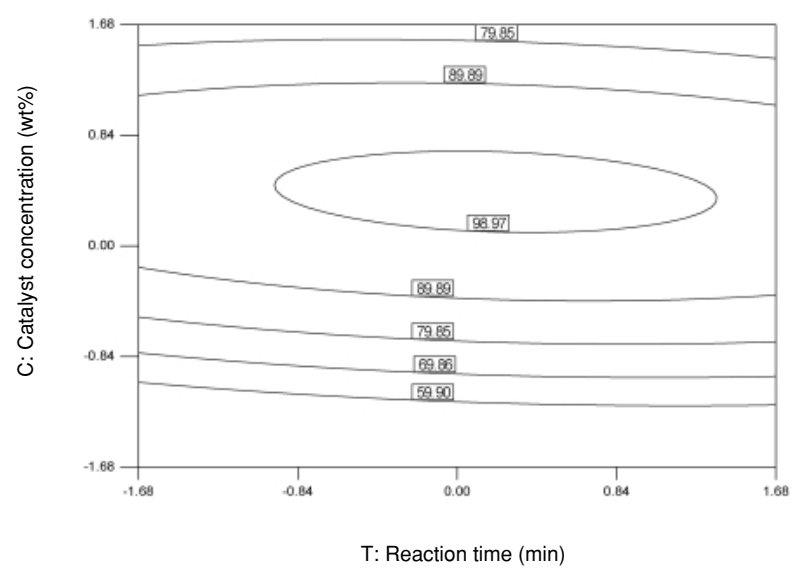

(B)

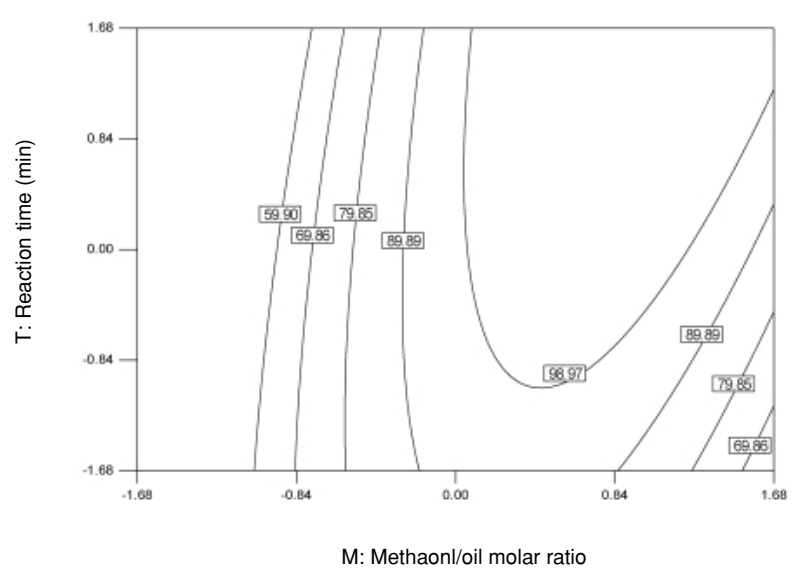

(C)

Fig. 1 Contour Plot of Methyl Ester Yield (wt\%) in Terms of Coded Factors: the Effect of Methanol/Oil Molar Ratio and Catalyst Concentration (A), Reaction Time and Catalyst Concentration (B), Methanol/Oil Molar Ratio and Reaction Time $(\mathbf{C})$ on Methyl Ester Production.

The third variable is held at zero level. 
Figure 1B showed the effect of reaction time and catalyst concentration on the methyl ester yield. At a certain level of catalyst concentration, there is no significant change in methyl ester yield when increasing the reaction time. Similar results were observed in Fig. $1 \mathrm{C}$ when the level of methanol/oil molar ratio was fixed. This could be explained by the higher $p$-value (0.6124) for the $T$ term in the model, indicating the non-significant effect. It could also be observed from Fig. 1B that when the $\mathrm{NaOH}$ catalyst concentration was about $1 \mathrm{wt} \%$, the methyl ester yield could be greater than $90 \mathrm{wt} \%$ in less than $5 \mathrm{~min}$. Compared with conventional mechanical stirring method, transesterifi-cation under ultrasonic irradiation was more efficient. This was confirmed by many researchers. Armenta et al. ${ }^{9}{ }^{9}$ observed that almost the entire transesterification process occurred within the first 10 min of treatment when using ultrasonic energy to transesterify fish oil in the presence of base catalysts. Stavarache et al. ${ }^{11)}$ concluded that the major part of the ultrasonically driven transesterification of vegetable oils under base catalysis took place in the first 3-10 min of reaction if not faster. The advantage of ultrasonic irradiation was attributed to the effect of cavitation, in which strong shock wave generated during the collapse of bubbles further disrupts the phase boundary, enhancing the mixing efficiency between immiscible triglycerides and alcohols.

\subsection{Attaining optimum conditions and model verification}

RIDGE analysis for maximization suggested the optimal values for the test variables in uncoded unit were as follows: reaction time $=8 \mathrm{~min}, \mathrm{NaOH}$ catalyst concentration $=1 \mathrm{wt} \%$, methanol/oil molar ratio $=6.2: 1$. Under the above optimum conditions of the variables, the model predicted that the maximum yield obtained could be $99 \mathrm{wt} \%$. Verification experiments were performed at the suggested optimal

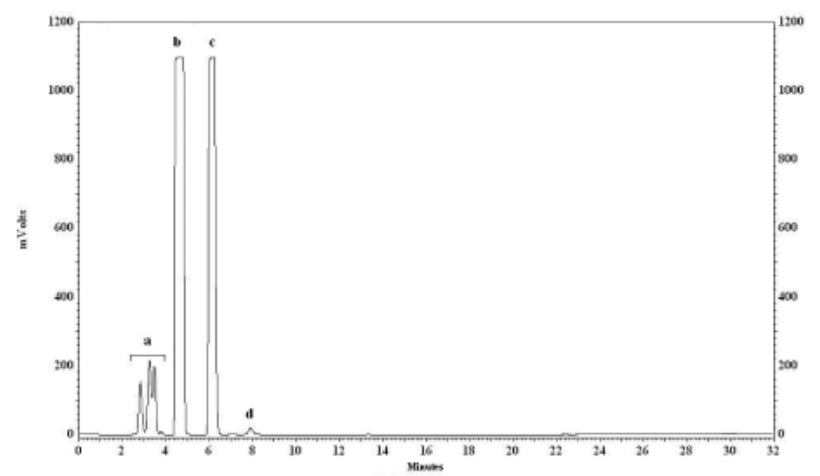

Fig. 2 HPLC Chromatogram of Methyl Ester. Lower case letters represent: a: monoglycrides, b: C18-2 (linoleic acid methyl ester), c: C18-1 (oleic acid methyl ester), d: C16-0 (palmitic acid methyl ester) conditions to examine the adequacy of the predicted model. The actual value was $98 \mathrm{wt} \%$ for the methyl ester yield and its HPLC chromatogram was shown in Fig. 2. Hence, the quadratic model was considered to be suitable to predict the methyl ester yield.

\subsection{The advantages of using ultrasonic irradiation to pro- duce biodiesel}

It could be clearly seen from RSM results that methyl ester produced by using ultrasonic irradiation exhibited many advantages. Compared with conventional mechanical stirring method ${ }^{20)}$, it could not only reduce the transesterification processing time, but also decrease reaction temperature due to the increased chemical activity in the presence of cavitation. These will reduce the biodiesel production costs and make biodiesel more competitive in price than diesel fuel.

\section{CONCLUSION}

In summary, RSM was proved to be a powerful tool for the optimization of methyl ester production under ultrasonic irradiation at room temperature. A second-order model was successfully developed to describe the relationships between methyl ester yield and test variables, including methanol/oil molar ratio, $\mathrm{NaOH}$ catalyst concentration and reaction time. The optimal conditions for the maximum methyl ester yield were found to be at methanol/oil molar ratio of 6.2:1, $\mathrm{NaOH}$ catalyst concentration of $1 \mathrm{wt} \%$ (by the weight of crude cottonseed oil), reaction time of 8 min. Validation experiment further confirmed the accuracy of the model. The transesterification process under ultrasonic irradiation could be more efficient, making biodiesel production more competitive in price than diesel fuel.

\section{ACKNOWLEDGEMENTS}

The authors are grateful to the Cotton Inc. and the Clemson University Vice President Research Fund for the financial support.

\section{References}

1. Lang, X.; Dalai, A.K.; Bakhshi, N.N.; Reaney, M.J.; Hertz, P.B. Preparation and characterization of biodiesels from various bio-oils. Bioresour. Technol. 80, 53-62 (2001).

2. Antolín, G.; Tinaut, F.V.; Briceño, Y.; Castaño, V.; Pérez, C.; Ramírez, A.I. Optimisation of biodiesel production by sunflower oil transesterification. Bioresour. Technol. 83, 111-114 (2002). 
3. Vicente, G.; Martínez, M.; Aracil, J. Integrated biodiesel production: a comparison of different homogeneous catalysts systems. Bioresour. Technol. 92, 297-305 (2004).

4. Hu, J.B.; Du, Z.X.; Tang, Z.; Min, E.Z. Study on the solvent power of a new green solvent: Biodiesel. Ind. Eng. Chem. Res. 43, 7928-7931 (2004).

5. Shieh, C.J.; Liao, H.F.; Lee, C.C. Optimization of lipasecatalyzed biodiesel by response surface methodology. Bioresour. Technol. 88, 103-106 (2003).

6. Colucci, J.A.; Borrero, E.E.; Alape, F. Biodiesel from an alkaline transesterification reaction of soybean oil using ultrasonic mixing. J. Am. Oil Chem. Soc. 82, 525-530 (2005).

7. Stavarache, C.; Vinatoru, M.; Maeda, Y. Ultrasonic versus silent methylation of vegetable oils. Ultrason. Sonochem. 13, 401-407 (2006).

8. Ji, J.B.; Wang, J.L.; Li, Y.C.; Yu, Y.L.; Xu, Z.C. Preparation of biodiesel with the help of ultrasonic and hydrodynamic cavitation. Ultrasonics 44, e411-414 (2006).

9. Armenta, R.E.; Vinatoru, M.; Burja, A.M.; Kralovec, J.A.; Barrow, C.J. Transesterification of fish oil to produce fatty acid ethyl esters using ultrasonic energy. $J$. Am. Oil Chem. Soc. 84, 1045-1052 (2007).

10. Stavarache, C.; Vinatoru, M.; Nishimura, R.; Maeda, Y. Fatty acids methyl esters from vegetable oil by means of ultrasonic energy. Ultrason. Sonochem. 12, 367-372 (2005).

11. Stavarache, C.; Vinatoru, M.; Maeda, Y. Aspects of ultrasonically assisted transesterification of various vegetable oils with methanol. Ultrason. Sonochem. 14, 380-386 (2007).
12. Hanh, H.D.; Dong, N.T.; Okitsu, K.; Maeda, Y.; Nishimura, R. Effects of molar ratio, catalyst concentration and temperature on transesterification of triolein with ethanol under ultrasonic irradiation. J. Jpn. Petrol. Inst. 50, 195-199 (2007).

13. Ghadge, S.V.; Raheman, H. Process optimization for biodiesel production from mahua (Madhuca indica) oil using response surface technology. Bioresour. Technol. 97, 379-384 (2006).

14. Tiwari, A.K.; Kumar, A.; Raheman, H. Biodiesel production from jatropha oil (Jatropha curcas) with high free fatty acids: An optimized process. Biomass Bioenerg. 31, 569-575 (2007).

15. Li, W.; Du, W.; Liu, D.H. Optimization of whole cell-catalyzed methanolysis of soybean oil for biodiesel production using response surface methodology. J. Mol. Catal. B-Enzym. 45, 122-127 (2007).

16. Yuan, X.Z.; Liu, J.; Zeng, G.M.; Shi, J.G.; Tong, J.Y.; Huang, G.H. Optimization of conversion of waste rapeseed oil with high FFA to biodiesel using response surface methodology. Renew. Energ. 33, 1678-1684 (2008).

17. AOCS official method Ca 5a-40. Free fatty acids. in Sampling and Analysis of Commercial Fats and Oils. AOCS press. Champaign (1997).

18. SAS / STAT ${ }^{\circledR}$ User's Guide, Version 8, Cary, NC, pp. 3029-3038 (1999).

19. Demirbaş, A. Biodiesel fuels from vegetable oils via catalytic and non-catalytic supercritical alcohol transesterifications and other methods: a survey. Energy Convers. Manage. 44, 2093-2109 (2003).

20. Vyas, A.P.; Verma, J.L.; Subrahmanyam, N. A review on FAME production processes. Fuel 89, 1-9 (2010). 